\title{
Occurrence of IL-1, IL-10, CD25, CD40, and CD69 in the tissue of palatine tonsils
}

\author{
Przemysław Bant ${ }^{1}$, Witold Owczarek², Kornel Szczygielski ${ }^{1}$, Szczepan Cierniak ${ }^{3}$, Joanna Kania ${ }^{3}$, Dariusz Jurkiewicz ${ }^{1}$
}

${ }^{1}$ Department of Otolaryngology with Division of Cranio-Maxillo-Facial Surgery, Military Institute of Medicine, Warsaw, Poland ${ }^{2}$ Department of Dermatology, Military Institute of Medicine, Warsaw, Poland

${ }^{3}$ Department of Pathomorphology, Military Institute of Medicine, Warsaw, Poland

Adv Dermatol Allergol 2022; XXXIX (1): 182-188

DOI: https://doi.org/10.5114/ada.2021.110285

\begin{abstract}
Introduction: Palatine tonsil disease often coexists with dermatological diseases. Correct diagnosis of inflammation of the palatine tonsil tissue and removal of the diseased palatine tonsils results in remission of the disease. Aim: To determine similarities and differences in the immunohistochemistry profile of the palatine tonsil tissue between tonsillitis and hypertrophy, including location of the immunohistochemistry reactions in specific histological sites.

Material and methods: A prospective analysis of 50 palatine tonsils that had undergone tonsillectomy due to tonsillitis (30 cases) and hypertrophy (20 cases) was performed. The collected material underwent immunohistochemistry staining for: IL-1, IL-10, CD25, CD40, and CD69, and subsequently phenotypic expression of the obtained results was performed including their histological location.

Results: Statistically significant differences $(p<0.05)$ between the tonsillitis and hypertrophy groups were found for almost all IHC reactions in the epithelium covering the tonsils for CD-25, CD-69, IL-1, IL-10. Furthermore, significant differences between these groups were found for IL-10 reaction in the subepithelial inflammatory infiltrate and follicular centres of lymphatic follicles as well as for CD-69 reaction between the follicles. When all the locations were summarized, significant $(p<0.05)$ differences were found for all IHC reactions except for CD-40.

Conclusions: The investigated markers and cytokines: CD25 and CD69, and IL-1 and IL-10 are more abundant in tonsillitis than in hypertrophy of the palatine tonsils.
\end{abstract}

Key words: interleukins, palatine tonsils, immunohistochemistry.

\section{Introduction}

Palatine tonsils are secondary lymphatic organs containing aggregates of lymphoid cells, and they belong to the mucosa-associated lymphoid tissue (MALT). The pharyngeal mucosa is equipped with a complex secretory immune system. B cells are stimulated by an antigen initially in the MALT regions, and thus formed stimulated lymphocytes migrate to the glandular sites, where they differentiate into Ig-producing cells [1]. White blood cells, mainly lymphocytes, are in all histological locations in the palatine tonsils, including the covering epithelium (they are called intraepithelial leukocytes - IEL). The IEL count in patients with tonsillitis is significantly higher than in patients with palatine tonsil hypertrophy. This is caused by a selective increase of the T-cell CD8 Vdelta1/ Vgamma9 population [2]. CD4+ T cells account for small- er share of IEL in the palatine tonsils than CD8+ cells; the former occur with B cells in the crypt epithelium [3]. $B$ cells occur mainly in 3 locations in the palatine tonsils: in the mantle zone of a lymphatic nodule, in the follicular centres, and as intraepithelial cells [4]. The epithelium that lines the crypts contains dendritic cells that can transport exogenous antigens to the extracellular areas of $T$ cells and to the B-cell vesicles [5]. Close association between the crypt epithelium and the lymphoid component persists throughout human life. Immune stimulation begins shortly after birth, at approximately 2 weeks of age, in response to exogenous antigens [6]. Multiple publications have shown that tonsillitis can induce or coexist with dermatological diseases and that tonsillectomy results in the disease remission. In 1935 Andrews and Machecek were the first to show that tonsillectomy

Address for correspondence: Przemysław Bant, Department of Otolaryngology with Division of Cranio-Maxillo-Facial Surgery, Military Institute of Medicine, Warsaw, Poland, e-mail: preston28@me.com

Received: 19.08.2021, accepted: 22.09.2021. 
was effective in the treatment of palmoplantar pustulosis (PPP) [7]. In the conducted study 9/24 PPP patients were cured after tonsillectomy. Another publication reported investigation of 124 PPP patients. The investigators showed that patients who underwent tonsillectomy exhibited a much higher cure rate than patients who received other therapies [8]. Yamakita et al. found that a group of patients who underwent tonsillectomy exhibited higher improvement rate of PPP skin lesions that the group who did not receive such a procedure. These results were further supported by a randomized prospective comparative study $[9,10]$.

In another publication the authors demonstrated similar correlations: 109 (94\%) of 116 patients and 52 (88\%) of 59 patients, who were assessed using a subjective self-assessment and the objective PPP Area and Severity Index (PPPASI), exhibited improvement of the PPP-induced skin lesions after tonsillectomy [11]. These data clearly show that palatine tonsil diseases may affect dermatological diseases and tonsillectomy results in reduction or complete resolution of skin lesions. Another authors [12] exhibited a positive correlation between the palatine tonsil disease and psoriasis. They showed that the risk of tonsillitis was higher in paediatric patients with psoriasis. The publication [13] reported Immunochip genotyping and their analysis. The investigations revealed that $\mathrm{HLA}-\mathrm{C}^{*} 06$ : 02 is an allele of risk for both palatine tonsillitis and psoriasis. The same 2 association peaks for $\sim 30-31.5 \mathrm{Mb}$ were associated with both these diseases. The authors of another study showed that tonsillectomy reduces signs and symptoms of Behcet's disease [14].

It seems that an increased activity of pro-inflammatory factors may take place in the affected palatine tonsils. CD25, coded by IL2RA, is one of them [15]. It is a transmembrane protein present in the activated lymphocytes, especially in Treg cells [16]. There are multiple mechanisms responsible for increased CD25 expression in $T$ cells. Binding of a mitogen or alloantigen by $T$ cells activates their receptors, causing inflow of the calcium ions, resulting in phosphorylation of protein kinase $C$ and triggering of a cascade of reactions resulting in an increased CD25 level (by 5- to 20-fold) [17]. CD25 expression by $T$ cells is additionally stimulated by IL-2 that induces a positive feedback loop through STAT5 [18].

A study enrolling patients with chronic tonsillitis qualified to tonsillectomy found CD25 cells in all patients [19]. Another study assessed activation of T cells in the palatine tonsils in PPP patients. Analysis of flow cytometry showed that the CD25+ cell count in the tonsils of PPP patients was significantly higher than in patients without the inflammatory process in their tonsils [20]. CD40 is another characteristic marker that is present on $B$ cells when they engage a specific ligand - CD154, present on activated T cells; it provides a signal required to initiate a humoral response. A cascade of immune response is triggered, re- sulting in activation of B cells and their maturation, differentiation and antibody production [21]. Palatine tonsils are an easily accessible and abundant source of human B cells from the secondary lymphatic tissue. They contain a heterogeneous mixture of B cells including several different phenotypes, such as naïve B cells, eGC, GC, and memory cells as well as plasmablasts [22]. CD69 may play an important role in chronic tonsillitis.

Persistence in a tissue environment depends on the ability of T cells to overwhelm exit signals. It can be achieved through expression of receptors that strengthen cellular interactions in the tissue and facilitate survival in a specific environment. The exit signal for T cells largely depends on S1P1 on T cells [23]. CD69 - a transmembrane type $C$ lectin - promotes persistence of lymphocytes in the tissues through binding to S1P1 receptor on their surface and its negative regulation [24, 25].

Another pathway that affects $T$ cell exit from the lymphatic system is CCR7 stimulation [26]. Its production is positively regulated by KLF2 and thus KLF2 blockade may affect CCR7-dependent lymphocyte migration [27]. The choice of CD69 as one of the pattern markers of the palatine tonsils is furthermore supported by a study that showed that CD69 expression was minimal on the circulating $T$ cells and accounted for $25-75 \%$ of CD8+ T cells in the spleen and tonsils [28].

Interleukin-1 (IL-1) also plays an important role. The IL-1 family plays a major role in modulating innate immunity and induction of inflammatory response [29]. This combination of 2 functions became obvious after the discovery that the cytoplasmic domain of IL-1 type I receptor is highly homologous with cytoplasmic domains of all Toll-like receptors (TLR) on the immune cells. Thus, developing inflammatory responses are induced both by activation of IL-1 ligands as well as TLR. While transduction of a signal of pro-inflammatory response induced by interleukin occurs through formation of a complex of type 1 IL-1 receptor (IL-1R1) and IL-1RAcP co-receptor [30], TLR induces inflammation through bacteria, microorganism products, viruses, nucleic acids, and damage-associated molecular patterns (DAMP) [31].

It must be emphasized that IL-10, known as a "cytokine synthesis inhibiting factor" (CSIF), is a potent cytokine with anti-inflammatory activity, produced mostly by subsets T cell lines (Treg, Th2). It can inhibit cytokine expression through effects on Th1 cell lines and can limit inflammatory response through effects on various immune cells [32]. IL-10 binds to a receptor (IL-10R) that is composed of two chains: IL-10R1 and IL-10R2. Association of IL-10 with IL-10R results in signal transduction through its main mediator - STAT3 - and regulation of inflammatory response [33]. It must be emphasized that a T-cell population producing large amounts of IL-10 and potently inhibiting immune response was identified in human palatine tonsils [34]. Two recent papers have also described the existence of IL-10 (a product of Tfh cells): one in which the cells ap- 
pear during chronic viral infection in mice [35] and another in which they were found in human tonsils [36].

\section{Aim}

As the above-mentioned data indicate, diagnosis of a disease of palatine tonsils is of utmost importance. Unfortunately, the proper clinical diagnosis is not always obvious, and its correct identification requires detailed, multifactorial investigations. Thus, the aim of the study was to assess the relationship between the clinical presentation and an immunohistochemistry phenotype of selected markers (including their histological locations) in the palatine tonsils resected due to tonsillitis and hypertrophy. We selected immunohistochemistry markers, the following titres of which are typical for inflammation: CD25, CD40, CD69, IL-1, and IL-10.

\section{Material and methods}

We analysed 50 palatine tonsils from tonsillectomy performed due to tonsillitis (30 cases) and palatine tonsil hypertrophy (20 cases) at the Department of Otolaryngology and Laryngological Oncology with Division of Cranio-Maxillo-Facial Surgery, Military Institute of Medicine, Warsaw, Poland. The patients were assigned to a specific group based on the previously reported qualification process [37]. Subsequently the collected material underwent immunohistochemistry testing. The exclusion criteria included patients with suspected neoplasm of the palatine tonsil and patients under 18 years of age.

Slides with immunohistochemistry reactions were scanned on a Pannoramic 250 FLASH 3DHISTECH scanner and evaluated under an Olympus BH63 light microscope, and subsequently the planned measurements were performed using 3DHISTECH CaseCenter ver. 2.7 software. Immunochemistry colour reaction was evaluated in 4 tonsillar regions, i.e. in the lymphatic follicles, crypt epithelium, interfollicular region, and subepithelial region. To report the phenotypic expression of immunohistochemistry reactions a specific assessment scale was used that included both staining intensity and percentage of stained cells; intensity of the immunohistochemistry reaction was assessed on a scale from 0 to 3 (none, weak, moderate, potent reaction), and the extent of the stained areas was assessed as follows: $<1 \%$ of cells $=0$; $1=1 ;>1-10=2 ; 11-33=3 ; 34-66=4 ;$ and $67-100=5$. Subsequently both values were added and thus the final score was achieved.

\section{Statistical analysis}

The data was analysed using GNU pspp 1.4 .1 software. The significance of mean values was assessed based on a single sample $t$ test. Significance of differences between parameters for 2 groups was determined based on a $t$ test for independent samples. Significance of differences between parameters for more than two groups was assessed using ANOVA with a post-hoc LSD Fisher test.

\section{Results}

Palatine tonsils were tested for 2 different cytokines and 3 lymphoid markers: IL-1, IL-10, CD25, CD40, and CD69 in each of the tested groups, including subdivision to specific histological locations in the palatine tonsils. Table 1 presents the results. Concurrent production of CD25, CD40, CD69, IL-1, and IL-10 was found in the palatine tonsillitis and hypertrophy. The measured average parameters were higher in the material obtained from the patients with tonsillitis than from the patients with palatine tonsil hypertrophy. When data for all the

Table 1. Results of the assessed cytokines and markers in the study groups, including subdivision into specific tissue structures of the palatine tonsils

\begin{tabular}{|c|c|c|c|c|c|c|c|}
\hline \multicolumn{2}{|c|}{ Variable } & \multirow{2}{*}{$\begin{array}{c}\text { Epithelial } \\
\text { Mean }\end{array}$} & \multirow{2}{*}{$\begin{array}{c}\text { Subepithelial } \\
\text { Mean }\end{array}$} & \multirow{2}{*}{$\begin{array}{c}\begin{array}{c}\text { Follicular } \\
\text { Mean }\end{array} \\
0.77\end{array}$} & \multirow{2}{*}{$\begin{array}{c}\begin{array}{c}\text { Follicular centre } \\
\text { Mean }\end{array} \\
2.37\end{array}$} & \multirow{2}{*}{$\begin{array}{c}\begin{array}{c}\text { Interfollicular } \\
\text { Mean }\end{array} \\
1.77\end{array}$} & \multirow{2}{*}{$\begin{array}{r}\text { Total } \\
\text { Mean } \\
2.37\end{array}$} \\
\hline \multirow{5}{*}{ 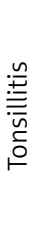 } & CD-25 & & & & & & \\
\hline & $C D-40$ & 1 & 1.67 & 0.07 & 1.47 & 0.47 & 0.84 \\
\hline & CD-69 & 2.1 & 3.33 & 0 & 1.73 & 1.73 & 1.78 \\
\hline & IL-1 & 3.2 & 4.43 & 0.13 & 2.27 & 3.4 & 2.69 \\
\hline & IL-10 & 0.97 & 1.13 & 0 & 0.7 & 0.5 & 0.7 \\
\hline \multirow{5}{*}{ 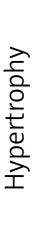 } & CD-25 & 0.55 & 2.3 & 0.55 & 1.75 & 1.25 & 1.28 \\
\hline & CD-40 & 0.3 & 0.9 & 0 & 1.1 & 0.35 & 0.53 \\
\hline & CD-69 & 0.95 & 2.85 & 0 & 2.1 & 0.4 & 1.26 \\
\hline & IL-1 & 0.85 & 4.2 & 0 & 1.25 & 3.15 & 1.89 \\
\hline & IL-10 & 0 & 0.1 & 0 & 0 & 0 & 0.02 \\
\hline
\end{tabular}

Statistically significant differences between the palatine tonsillitis and hypertrophy are marked in bold. 
Table 2. Comparison of assessed markers and cytokines in the group of chronic palatine tonsillitis, including specific tissue regions of the palatine tonsils

\begin{tabular}{|c|c|c|c|c|c|c|c|c|c|c|c|c|c|c|c|c|c|c|c|c|c|c|c|c|c|}
\hline \multirow[t]{2}{*}{ Variable } & \multicolumn{5}{|c|}{ CD-25 } & \multicolumn{5}{|c|}{ CD-40 } & \multicolumn{5}{|c|}{ CD-69 } & \multicolumn{5}{|c|}{ IL-1 } & \multicolumn{5}{|c|}{ IL-10 } \\
\hline & 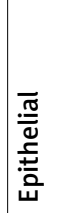 & 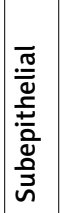 & 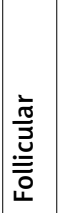 & 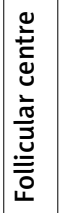 & 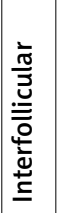 & 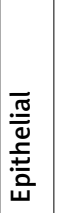 & 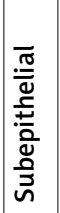 & 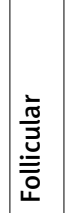 & 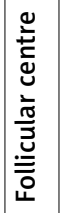 & 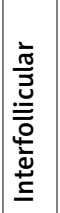 & 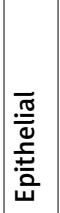 & 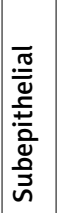 & $\begin{array}{l}\frac{\bar{\sigma}}{3} \\
\frac{3}{3} \\
\frac{\overline{0}}{4}\end{array}$ & 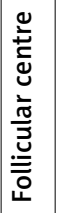 & 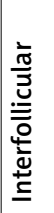 & 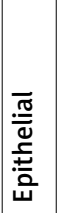 & 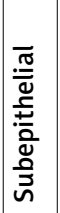 & 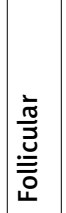 & 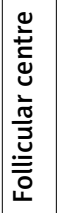 & 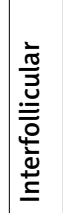 & 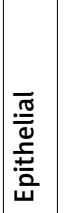 & 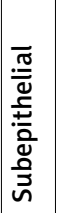 & 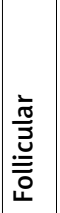 & 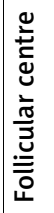 & 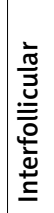 \\
\hline Epithelial & & & $x$ & & & & $x$ & $x$ & & & & $x$ & $x$ & & & & $x$ & $x$ & $x$ & & & & $x$ & & \\
\hline Subepithelial & & & $x$ & & & $x$ & & $x$ & $x$ & $x$ & $x$ & & $x$ & $x$ & $x$ & $x$ & & $x$ & $x$ & $x$ & & & $x$ & & \\
\hline Follicular & $x$ & $x$ & & $x$ & $x$ & $x$ & $x$ & & $x$ & & $x$ & $x$ & & $x$ & $x$ & $x$ & $x$ & & $x$ & $x$ & $x$ & $x$ & & & \\
\hline Follicular centre & & & $x$ & & & & $x$ & $x$ & & & & $x$ & $x$ & & & $x$ & $x$ & $x$ & & $x$ & & & & & \\
\hline Interfollicular & & & $x$ & & & & $x$ & & & & & $x$ & $x$ & & & & $x$ & $x$ & $x$ & & & & & & \\
\hline
\end{tabular}

Statistically significant differences for a specific marker for individual regions of the palatine tonsil are marked by an " $X$ ".

Table 3. Comparison of assessed markers and cytokines in the group of palatine tonsil hypertrophy including specific tissue regions of the palatine tonsils

\begin{tabular}{|c|c|c|c|c|c|c|c|c|c|c|c|c|c|c|c|c|c|c|c|c|c|c|c|c|c|}
\hline \multirow[t]{2}{*}{ Variable } & \multicolumn{5}{|c|}{ CD-25 } & \multicolumn{5}{|c|}{ CD-40 } & \multicolumn{5}{|c|}{ CD-69 } & \multicolumn{5}{|c|}{ IL-1 } & \multicolumn{5}{|c|}{ IL-10 } \\
\hline & 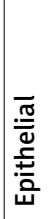 & 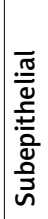 & 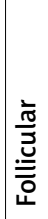 & 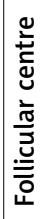 & 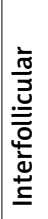 & 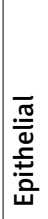 & 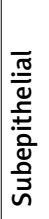 & 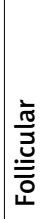 & 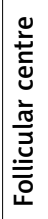 & 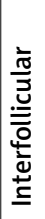 & 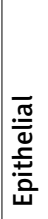 & 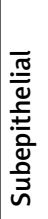 & 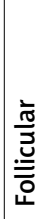 & 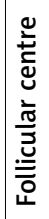 & 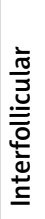 & 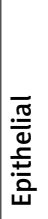 & 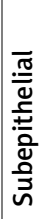 & 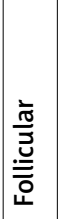 & 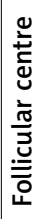 & 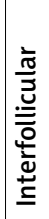 & 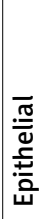 & 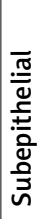 & 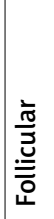 & 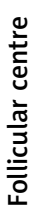 & 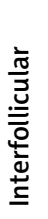 \\
\hline Epithelial & & $x$ & & $x$ & & & & & $x$ & & & $x$ & & $x$ & & & $x$ & & & $x$ & & & & & \\
\hline Subepithelial & $x$ & & & & $x$ & & & $x$ & & & $x$ & & $x$ & & $x$ & $x$ & & $x$ & $x$ & $x$ & & & & & \\
\hline Follicular & & & & & & & $x$ & & $x$ & & & $x$ & & $x$ & & & $x$ & & $x$ & $x$ & & & & & \\
\hline Follicular centre & $x$ & & & & & $x$ & & $x$ & & & $x$ & & $x$ & & $x$ & & $X$ & $x$ & & $x$ & & & & & \\
\hline Interfollicular & & $x$ & & & & & & & & & & $x$ & & $x$ & & $x$ & $x$ & $X$ & $x$ & & & & & & \\
\hline
\end{tabular}

Statistically significant differences for a specific marker for individual regions of the palatine tonsil are marked by an " $X$ ".

locations were summarized, significant $(p<0.05)$ differences were found for all reactions except for CD-40. The covering epithelium was the region exhibiting the most statistically significant differences between the tested markers, while no statistically significant differences were found between the tested groups in the follicular region. Furthermore, comparison of specific markers in the tonsillectomy group with regard to histological location in the tested palatine tonsils revealed that the most statistically significant differences were found for IL-1 and CD69, and the least for IL-10. On the other hand, the hypertrophy group exhibited statistically significant differences for the tested markers with the highest titres also for IL-1 and CD69, and the lowest for IL-10, for which no statistically significant correlation was found for this marker. Both the highest and lowest measured values for a specific marker for each compartment of the palatine tonsil tissue were higher in the tonsillitis than in the hypertrophy group. Tables 2 and 3 present the results.

\section{Discussion}

The study by Mikola et al. [38] found IL-10 in the palatine tonsil tissue affected by inflammation. Its average value was lower than in the hypertrophic palatine tonsil. We found the opposite relation in our study. We obtained higher value for the tonsillitis than for the hypertrophy. However, unlike our study, the authors of that paper did not identify a region of the palatine tonsil with the highest concentration of this cytokine. Moreover, the revealed differences did not reach statistical significance. We performed such a subdivision in our study and found the highest concentration of this cytokine in the subepithelial inflammatory infiltrate for 2 groups of palatine tonsils equal to 1.13 for the tonsillitis tissue and 0.1 for the 
hypertrophic palatine tonsils. As the authors emphasize, among the tested cytokines only the newly discovered anti-inflammatory cytokine, IL-37, was independently associated with the palatine tonsil hypertrophy, exhibiting slightly more potent anti-inflammatory response in these patients. Furthermore, the authors suggest that the palatine tonsil hypertrophy may be a consequence of chronic tonsillitis, suggesting blurring of the borders between the cytokine levels (e.g. IL-10) in both these conditions.

Huang et al. [39] found IL-1 and IL-10 in the hypertrophic palatine tonsil tissue as well as in hypertrophy with coexisting tonsillitis. The researchers demonstrated higher expression levels of the tested cytokines in the group with isolated hypertrophy. In our study we found higher expression of both IL-1 and IL-10 in the tonsillitis compared to hypertrophy tissue. Therefore, we obtained opposite relations in our study. As the authors of this publication emphasize, the aetiology of hypertrophy of the tonsillar lymphatic tissues remains unknown. They found high expression of VP1 in the hypertrophic palatine tonsils, indicating that the hypertrophy of the palatine tonsils could have been caused by a viral infection. Furthermore, the authors analysed immune mechanisms involved in the response triggered by the virus. The available literature proved that TLR recognizes the virus and initiates a series of cellular antiviral responses through intracellular signalling pathways $[40,41]$. The results obtained by these authors indicated that TLR4 and TLR7 were involved in the innate immune response triggered by viruses in isolated hypertrophic palatine tonsils. The different results obtained by us and authors of the above-mentioned publication may result from the lack of a homogeneous tonsillitis group. We believe that comparing cytokine profiles in 2 groups, one of which has the same component as the other, is imprecise and carries a high risk of error. We identified 2 independent groups in our study, achieving statistically significant results.

Geißler et al. [42] investigated the ability of T cells in tonsillitis to exhibit continuous high basic expression level of the surface CD25, CD69, and CD154 (CD40) in freshly isolated $T$ cells from the palatine tonsils. The results obtained in patients with tonsillitis indicated higher CD25, CD69, and CD154 (CD40) expression versus patients with peritonsillar abscess and palatine tonsil hypertrophy. We obtained compatible results, but statistically significant differences were obtained for all except the CD40 titre. CD25, CD40, and CD69 titres for the palatine tonsillitis group were $2.37,0.84$, and 1.78 , respectively, while CD25, CD40, and CD69 titres for the palatine tonsil hypertrophy group were $1.28,0.53$, and 1.26 , respectively. We also identified regions of the palatine tonsil characterized by expression of the above-mentioned markers. The highest values were obtained in the subepithelial region for CD25 and CD69 in both study groups and for CD40 in the subepithelial region in the tonsillitis group and the follicular centre for the hypertrophy group. As the authors of this publication emphasize, T cells in the tonsillitis exhibit signs of elevated basal activation status, mirroring high basal surface expression of, among others, CD69. This is compatible with the view that T cells in the tonsils are exposed to chronic stimulation. However, chronic exposure to an antigen, initially favouring accumulation of pathogen-specific effector T cells, may eventually result in exhaustion of effector T cells [43-46]. Finally, this results in high basal CD69 expression, associated with exhausted T cell phenotype [45, 47, 48].

Chen et al. [49] compared 2 groups of children: one group with obstructive sleep apnoea (OSA) syndrome without tonsillitis (OSAS group) and a control group that included children with tonsillitis without an accompanying OSA. They found higher IL-1 expression in the control group than in the palatine tonsil hypertrophy group. Moreover, IL-1 expression in the palatine tonsil tissue was higher in the core than in the cortex. We found the following IL-1 levels in the tonsillitis group in the specific palatine tonsil zones (epithelial-3.2, subepithelial -4.43, follicular - 0.13, follicular center -2.27 , interfollicular 3.4). In our analysis its level was also higher in the tonsillitis group. The authors of the publication compared IL10 concentrations between the study groups. The OSAS group exhibited higher IL-10 levels than the control group. Our analysis provided the opposite results. Agren et al. [50] also assessed cytokines in the hypertrophic palatine tonsils with severe OSAS without an accompanying infection and palatine tonsils with recurrent infections. The IL-1 level was significantly higher in the tonsillitis group than in the hypertrophy group. This supports our results. The discussed study [49] and our study included palatine tonsils, excluding the adenoid, which also played an important role in the OSA pathophysiology and was associated with inflammatory markers related to sleep disorders. Moreover, the authors examined only a paediatric population aged from 3 to 12 years. Furthermore, the study enrolled only 34 patients. Our study group was more varied with regard to age and number of cases. This could contribute to different results for IL-10 between the study groups.

In another study [51] the authors analysed effects of adenotonsillectomy on the evolution of inflammatory markers in patients with palatine tonsillitis, palatine tonsil hypertrophy, and the 2 combined. They found reduction of IL-1 and IL-10 levels after tonsillectomy and higher levels of these cytokines in the tonsillectomy group versus the control group. In the performed study the authors achieved higher IL-10 levels in the inflammation group versus the hypertrophy group (OSAS). These relations are compatible with our results. Nevertheless, IL-1 values obtained by the investigators were higher in the OSAS group versus the tonsillectomy group. These results contradict ours. According to the authors of this publication, the higher IL-10 titre in the tonsillitis group might have resulted from pathophysiological changes induced by 
the palatine tonsil infection. This has a 2-pronged effect on the inflammation of the palatine tonsil, both through production of pro-inflammatory cytokines and through increased levels of the anti-inflammatory proteins that counteract it. The reversibility of this process with tonsillectomy supports the concept of restoration of balance that is lacking.

\section{Conclusions}

The current results indicate similarities and differences in the cytokine pattern and distribution of the lymphoid cells in the palatine tonsil tissue in the 2 defined diseases. Moreover, highly compartmentalized cytokine production and the presence of lymphoid cells can be found. The highest concentrations of IL-1, IL-10, CD25, and CD69 were found in both palatine tonsil groups in the region of the subepithelial inflammatory infiltrate, while CD40 was found in the tonsillitis group in the region of the subepithelial inflammatory infiltrate and in the hypertrophy group in the follicular centre. Increased activity of these factors favours persistence of chronic palatine tonsillitis, which may affect local and systemic diseases, including dermatological disorders. The investigated markers and cytokines CD25, CD69, IL-1, and IL-10 are more abundant in the palatine tonsillitis than in hypertrophy. The obtained results are statistically significant $(p<0.05)$. Thus, tonsillitis potentially underlies systemic changes and should preferably be eliminated. The presence of the investigated markers and cytokines in all parts of the palatine tonsils must be emphasized. This indicates the requirement for resection of the whole palatine tonsil rather than its partial resection.

\section{Conflict of interest}

The authors declare no conflict of interests.

\section{References}

1. Yong-Jun L, Christophe A. Follicular center development. Immunol Rev 1997; 156: 111-26.

2. Olofsson K, Hellström S, Hammarström ML. The surface epithelium of recurrent infected palatine tonsils is rich in gammadelta T cells. Clin Exp Immunol 1998; 111: 36-47.

3. Graeme-Cook F, Bhan AK, Harris NL. Immunohistochemical characterization of intraepithelial and subepithelial mononuclear cells of the upper airways. Am J Pathol 1993; 143: 1416-22.

4. Dono M, Burgio VL, Tacchetti C, et al. Subepithelial B cells in the human palatine tonsil. I. Morphologic, cytochemical and phenotypic characterization. Eur J Immunol 1996; 26: 2035-42.

5. Seungkoo L, Kyungho C, Hanjong A, et al. TuJ1 (class III betatubulin) expression suggests dynamic redistribution of follicular dendritic cells in lymphoid tissue. Eur J Cell Biol 2005; 84: 453-9.
6. Stephen W, Pam N, Karin H, et al. Development of the palatine tonsil in conventional and germ-free piglets. Dev Comp Immunol 2005; 29: 977-8.

7. Andrews GC, Machacek GF. Pustular bacterids of the hand and feet. Arch Dermatol Syphilol 1935; 32: 837-47.

8. Ono T, Jono M, Kito M, et al. Evaluation of tonsillectomy as a treatment for pustulosis palmaris et plantaris. Acta Otolaryngol Suppl 1983; 401: 12-6.

9. Yamakita T, Washimi Y, Yagami M, et al. Clinical effect of tonsillectomy in patients with pustulosis palmaris et plantaris (PPP). Jpn J Dermatol 2004; 114: 2319-26.

10. Yamakita T, Shimizu Y, Naito K, et al. Clinical effect of tonsillectomy in patients with pustulosis palmaris et plantaris (PPP). Stomatopharyngology 2009; 22: 49-54.

11. Takahara M. Clinical outcome of tonsillectomy for palmoplantar pustulosis and etiological relationship between palmoplantar pustulosis and tonsils. Adv Otorhinolaryngol 2011; 72: 86-8.

12. Groot J, Blegvad C, Nybo Andersen AM, et al. Tonsillitis and pediatric psoriasis: cohort and cross-sectional analyses of offspring from the Danish National Birth Cohort. J Am Acad Dermatol 2020; 82: 666-74.

13. Haapasalo K, Koskinen L, Suvilehto J, et al. The psoriasis risk allele HLA-Cw0602 shows evidence of association with chronic or recurrent streptococcal tonsillitis. Infect Immun 2018; 86: e00304-18.

14. Nanke Y, Kobasigawa T, Yoda K, et al. Tonsillectomy to effectively treat a patient with Behçet's disease. Intern Med 2016; 55: 515-7.

15. Leonard WJ, Donlon TA, Lebo RV, et al. Localization of the gene encoding the human interleukin-2 receptor on chromosome 10. Science 1985; 228: 1547-9.

16. Sakaguchi S, Sakaguchi N, Asano M, et al. Immunologic selftolerance maintained by activated T cells expressing IL-2 receptor alpha-chains (CD25). Breakdown of a single mechanism of self-tolerance causes various autoimmune diseases. J Immunol 1995; 155: 1151-64.

17. Waldmann TA. Anti-Tac (daclizumab, Zenapax) in the treatment of leukemia, autoimmune diseases, and in the prevention of allograft rejection: a 25-year personal odyssey. J Clin Immunol 2007; 27: 1-18.

18. Boyman O, Sprent J. The role of inter- leukin-2 during homeostasis and activation of the immune system. Nat Rev Immunol 2012; 12: 180-90.

19. Simark-Mattsson C, Dahlgren U, Roos K. CD4+CD25+ T lymphocytes in human tonsils suppress the proliferation of CD4+CD25- tonsil cells. Scand J Immunol 2002; 55: 606-11.

20. Takahara M, Kishibe K, Nozawa H, et al. Increase of activated T-cells and up-regulation of Smad7 without elevation of TGF-beta expression in tonsils from patients with pustulosis palmaris et plantaris. Clin Immunol 2005; 115: 192-9.

21. Miga A, Masters S, Gonzalez M, et al. The role of CD40CD154 interactions in the regulation of cell mediated immunity. Immunol Invest 2000; 29: 111-4.

22. Jackson SM, Wilson PC, James JA, et al. Human B cell subsets. Adv Immunol 2008; 98: 151-224.

23. Schwab SR, Cyster JG. Finding a way out: lymphocyte egress from lymphoid organs. Nat Immunol 2007; 8: 1295-301.

24. Shiow LR, Rosen DB, Brdicková N, et al. CD69 acts downstream of interferon- alpha/beta to inhibit S1P1 and lymphocyte egress from lymphoid organs. Nature 2006; 440: 540-4.

25. Mackay LK, Braun A, Macleod BL, et al. Cutting edge: CD69 interfer- ence with sphingosine-1-phosphate receptor func- 
tion regulates peripheral T cell retention. J Immunol 2015; 194: 2059-63.

26. Pham THM, Okada T, Matloubian M, et al. S1P1 receptor signaling overrides retention mediated by $\mathrm{G}$ alpha i-coupled receptors to promote T cell egress. Immunity 2008; 28: 122-33.

27. Carlson CM, Endrizzi BT, Wu J, et al. Kruppel-like factor 2 regulates thymocyte and T-cell migration. Nature 2006; 442 : 299-302.

28. Sathaliyawala T, Kubota M, Yudanin N, et al. Distribution and compartmentalization of human circulating and tissueresident memory T cell subsets. Immunity 2013; 38: 187-97.

29. Dinarello CA. Immunological and inflammatory functions of the inter- leukin-1 family. Annu Rev Immunol 2009; 27 : 519-50.

30. Garlanda C, Dinarello CA, Mantovani A. The interleukin-1 family: back to the future. Immunity 2013; 39: 1003-18.

31. Dinarello CA. Overview of the IL-1 family in innate inflammation and acquired immunity. Immunol Rev 2018; 281: 8-27.

32. Fiorentino DF, Bond MW, Mosmann TR. Two types of mouse Thelper cell. IV. Th2 clones secrete a factor that inhibits cytokine production by Th1 clones. J Exp Med 1989; 170: 2081-95.

33. Moore KW, de War Malefyt R, Coffman RL, et al. Interleukin-10 and the interleukin-10 receptor. Annu Rev Immunol 2001; 19: 683-765.

34. Sumitomo S, Nakachi S, Okamura T, et al. Identification of tonsillar CD4+ CD25- $\mathrm{LAG3}^{+} \mathrm{T}$ cells as naturally occurring IL10-producing regulatory $T$ cells in human lymphoid tissue. J Autoimmun 2017; 76: 75-84.

35. Xin G, Zander R, Schauder DM, et al. Single-cell RNA sequencing unveils an IL-10-producing helper subset that sustains humoral immunity during persistent infection. Nat Commun 2018; 9: 5037.

36. Canete PF, Sweet RA, Gonzalez-Figueroa P, et al. Regulatory roles of IL-10-producing human follicular T cells. J Exp Med 2019; 216: 1843-56.

37. Bant P, Szczygielski K, Cierniak S, et al. Tonsillectomy in own material. Otolaryngol Pol 2020; 74: 1-5.

38. Mikola E, Elenius V, Saarinen M, et al. Tonsillar cytokine expression between patients with tonsillar hypertrophy and recurrent tonsillitis. Clin Transl Allergy 2018; 8: 22.

39. Huang Q, Hua H, Li W, et al. Simple hypertrophic tonsils have more active innate immune and inflammatory responses than hypertrophic tonsils with recurrent inflammation in children. J Otolaryngol Head Neck Surg 2020; 49: 35.

40. Kurt-Jones EA, Popova L, Kwinn L, et al. Pattern recognition receptors TLR4 and CD14 mediate response to respiratory syncytial virus. Nat Immunol 2000; 1: 398-401.

41. Bowie A, Kiss-Toth E, Symons JA, et al. A46R and A52R from vaccinia virus are antagonists of host IL-1 and toll-like receptor signaling. Proc Natl Acad Sci USA 2000; 97: 10162-7.

42. Geißler K, Markwart R, Requardt RP, et al. Functional characterization of T-cells from palatine tonsils in patients with chronic tonsillitis. PLoS One 2017; 12: e0183214.

43. Wherry EJ, Barber DL, Kaech SM, et al. Antigen-independent memory CD8 T cells do not develop during chronic viral infection. Proc Natl Acad Sci USA 2004; 101: 16004-9.

44. Zajac AJ, Vance RE, Held W, et al. Impaired anti-viral T cell responses due to expression of the Ly49A inhibitory receptor. I Immunol 1999; 163: 5526-34.

45. Zajac AJ, Blattman JN, Murali-Krishna K, et al. Viral immune evasion due to persistence of activated T cells without ef fector function. J Exp Med 1998; 188: 2205-13.

46. Wherry EJ, Blattman JN, Murali-Krishna K, et al. Viral persistence alters CD8 T-cell immunodominance and tissue dis- tribution and results in distinct stages of functional impairment. J Virol 2003; 77: 4911-27.

47. Yi JS, Cox MA, Zajac AJ. T-cell exhaustion: characteristics, causes and conversion. Immunology 2010; 129: 474-81.

48. Fuller MJ, Khanolkar A, Tebo AE, et al. Maintenance, loss, and resurgence of T cell responses during acute, protracted, and chronic viral infections. J Immunol 2004; 172: 4204-14.

49. Chen VG, Guerreiro da Fonseca VM, Amaral JB, et al. Inflammatory markers in palatine tonsils of children with obstructive sleep apnea syndrome. Braz J Otorhinolaryngol 2020; 86: 23-9.

50. Agren K, Lindberg K, Samulesson A, et al. What is wrong in chronic adenoiditis/tonsillitis immunological factor. Int J Pediatr Otorhinolaryngol 1999; 49 Suppl 1: S137-9.

51. Marcano-Acuńa ME, Carrasco-Llatas M, Tortajada-Girbés M, et al. Impact of adenotonsillectomy on the evolution of inflammatory markers. Clin Otolaryngol 2019; 44: 983-8. 